BGU-PH-95/06, CERN-TH/95-144

\title{
Relic Gravitational Waves from String Cosmology
}

\author{
R. Brustein ${ }^{(a, c)}$, M. Gasperini ${ }^{(b)}$, M. Giovannini ${ }^{(b, c)}$ and G. Veneziano ${ }^{(c)}$ \\ (a) Department of Physics, Ben-Gurion University, Beer-Sheva 84105, Israel \\ (b) Dipartimento di Fisica Teorica, Via P.Giuria 1, 10125 Turin, Italy \\ (c) Theory Division, CERN, CH-1211, Geneva 23, Switzerland
}

\begin{abstract}
A large class of string-cosmology backgrounds leads to a spectrum of relic stochastic gravitational waves, strongly tilted towards high frequencies, and characterized by two basic parameters of the cosmological model. We estimate the required sensitivity for detection of the predicted gravitational radiation and show that a region of our parameter space is within reach for some of the planned gravitational-wave detectors.
\end{abstract}

CERN-TH/95-144

June 1995 


\section{Introduction}

It is notoriously difficult to find accessible experimental signatures of fundamental strings because of their small Planckian size. Possibly, an interesting exception to this rule is represented by the cosmological predictions of string theory, since these originate from physics of the Early-Universe, when space-time curvatures may have been of Planckian strength.

In order to arrive at some concrete, yet generic, predictions of string cosmology, we shall consider a large class of models in which a period of dilaton-driven inflation [1, 2, 3] is followed by a stringy epoch, during which the curvature remains of the order of the string scale $\lambda_{s}^{-2}$, and then finally, after possibly a short dilaton-relaxation era, by the standard (radiation then matter dominated) cosmology. As discussed in detail in [4, the presence of a high-curvature stringy epoch appears to be unavoidable for a viable inflationary string cosmology scenario. Additional support to this point of view was given in [5].

Recently, in collaboration with V. Mukhanov [6], we have discussed the main properties of metric perturbations in a dilaton-driven background. Particular attention was given to the correct treatment of scalar perturbations which, in a standard treatment, appear to grow too large for the applicability of linear perturbation theory. By carefully "gauging down" certain growing modes, we were able to show that both scalar and tensor perturbations can be treated perturbatively and that they exhibit very similar spectra. A characteristic feature of these spectra is that, unlike the spectra of the standard inflationary scenarios, they are not flat but strongly tilted towards higher frequencies, as originally noted in [0]].

In this note we will concentrate on tensor perturbations, those associated with gravitational waves $(\mathrm{GW})$, at frequencies which may be accessible to earth-based GW detectors.

Quite amazingly, their spectrum turns out to be rather independent of most of the details of the above string cosmology scenario. As shown in the first part of this paper, the spectrum can be completely given in terms of two parameters, the value $g_{s}$ of the string coupling parameter at the end of the dilaton-driven phase (equivalently, at the beginning of the string 
epoch), and the total red-shift $z_{s}$ occurring during the string era. The theoretical advantage of considering GW signals stems from the fact that, unlike the electromagnetic perturbations which underwent a complicated history until recombination, gravitons decoupled since right after the Planck era. As a consequence, their present spectrum should be a faithful portrait of the very early Universe. On the other hand, the detection of a cosmological background of GW requires extremely precise length measurements, typically at least of the order of $\delta L / L<10^{-21}[8]$. In the second part of this paper we will show that the expected range of $g_{s}$ and $z_{s}$ includes a region which should be accessible to future gravitational wave experiments.

Throughout this paper we shall be working in the so-called String-frame, in which weakly interacting strings move along geodesic surfaces. Identical results would follow by adopting the more conventional Einstein frame in which the curvature is canonically normalized, but we believe the physics to be more transparent in the former frame. In the String frame the string length parameter which is the short-distance cut-off of string theory, $\lambda_{s}=\sqrt{\alpha^{\prime} \hbar}$, is constant, while the Planck length, $\lambda_{P}=\sqrt{G_{N} \hbar}$, evolves in time as $\lambda_{P}=e^{\phi / 2} \lambda_{s}$ in a timedependent dilaton background. In our scenario, the background evolution starts from the string perturbative vacuum [3], therefore $\lambda_{P}$ grows from a very small initial value to a value $g_{s} \lambda_{s}$ reached at the beginning of the stringy era and, finally, to its present (very large!) value of about $10^{-33} \mathrm{~cm}$ at the beginning of the radiation dominated era.

\section{Primordial gravitational-wave spectra}

Let us consider, for the moment, an isotropic, $(3+1)$-dimensional, spatially flat cosmology. Following [9] (see also [6]) it is easy to show that the canonical variable $\psi$ associated with tensor perturbations is related to the String-frame metric $g_{\mu \nu}$ via

$$
g_{\mu \nu}=a^{2}\left(\eta_{\mu \nu}+h_{\mu \nu}\right)=a^{2}\left(\eta_{\mu \nu}+\frac{g}{a} \psi_{\mu \nu}\right),
$$

where $a(t)$ is the isotropic scale factor, $\eta_{\mu \nu}$ is the flat Minkowski metric and $g=\exp (\phi / 2)$. The Fourier modes of each of the two physical, transverse-traceless, polarizations satisfy the 
following simple wave equation [9]

$$
\psi_{k}^{\prime \prime}+\left[k^{2}-V(\eta)\right] \psi_{k}=0, \quad V(\eta)=(g / a)(a / g)^{\prime \prime}
$$

where a prime denotes differentiation with respect to conformal time $\eta(a d \eta \equiv d t)$ and $k$ is the comoving wave number, related to the physical one, $\omega$, by $k=\omega a$. Note that, since $a / g$ has power-like behavior in $\eta$ during the dilaton-driven phase [1, 2, [3], $V(\eta)$ grows like $\eta^{-2}$ during that epoch reaching a maximum at $\eta=\eta_{s}$, i.e. when $H^{-1} \sim \eta_{s} a_{s} \sim \lambda_{s} \equiv M_{s}^{-1}$. We expect $V(\eta)$ to keep growing during the stringy era and then to fall rapidly to zero at the onset $\left(\eta=\eta_{1}\right)$ of the radiation-dominated era since, in that phase, $a / g \sim \eta$.

A given mode $k$ will be well inside the horizon initially, then hit the potential barrier $V(\eta)$ at some "exit" time $\eta_{e x} \sim k^{-1}$, and leave the barrier at some later "reentry" time $\eta=\eta_{r e} \sim \eta_{1}$. The approximate solutions of eq. (2.2) in these three regimes are given by

$$
\begin{gathered}
\psi_{k}=\frac{\lambda_{s}}{\sqrt{k}} e^{-i k \eta}, \quad \eta<\eta_{e x} \\
\psi_{k}=\frac{a}{g}\left[A_{k}+B_{k} \int^{\eta} d \eta^{\prime}\left(\frac{g}{a}\right)^{2}\right], \eta_{e x}<\eta<\eta_{r e} \\
\psi_{k}=\frac{\lambda_{s}}{\sqrt{k}}\left[c_{+}(k) e^{-i k \eta}+c_{-}(k) e^{i k \eta}\right], \quad \eta>\eta_{r e}
\end{gathered}
$$

Equation (2.3) enforces the proper normalization of the primordial vacuum fluctuations. In the regime described by eq. (2.4) the perturbation is frozen outside the horizon and the two terms appearing there correspond to the freezing of $h$ and of its canonically conjugate momentum, respectively. Finally, in (2.5), the magnitude of $c_{-}$(so-called Bogoliubov coefficient) gives the amplification of the GW with respect to a minimal vacuum fluctuation. The actual value of $\left|c_{-}\right|$can be easily obtained by matching the above solutions and their first derivatives at each transition time,

$$
2 k \eta_{1}\left|c_{-}(k)\right| \simeq \frac{g_{e x} / a_{e x}}{g_{r e} / a_{r e}}\left[1+k \eta_{1}\left(\frac{g_{r e} / a_{r e}}{g_{e x} / a_{e x}}\right)^{2}+k\left(g_{e x} / a_{e x}\right)^{-2} \int_{\eta_{e x}}^{\eta_{1}} d \eta(g / a)^{2}\right]
$$

At this point we have to insert some information about the background evolution. In the simple case at hand of a $D=3+1$ isotropic cosmology with static extra dimensions, the 
dilaton-driven inflationary background is simply given by [3]

$$
a(\eta)=(-\eta)^{-\frac{1}{1+\sqrt{3}}}, \quad \phi(\eta)=-\sqrt{3} \ln (-\eta), \quad a / g \sim(-\eta)^{1 / 2}, \quad-\infty<\eta<0
$$

while, for the string era, we will assume that $H$ and $\partial_{t} \phi$ are approximately constant and of order $\lambda_{s}^{-1}$. Limiting our attention for the time being to those scales which crossed the horizon during the dilaton-driven phase, we thus arrive at the following estimate

$$
2 k \eta_{1}\left|c_{-}(k)\right| \simeq\left(k / k_{s}\right)^{1 / 2} z_{s}\left(g_{s} / g_{1}\right)\left[1+z_{s}^{-3}\left(g_{1} / g_{s}\right)^{2}+\ln \left(k_{s} / k\right)+I\right], \quad k<k_{s}
$$

where we have denoted for convenience $a_{r e} / a_{s} \simeq a_{1} / a_{s}=z_{s}$. In eq. (2.8) $k_{s}^{-1} \sim \eta_{s} \sim\left(H_{s} a_{s}\right)^{-1}$ is the last scale exiting during the dilaton-driven phase, $g_{1}=\exp \left(\phi_{1} / 2\right)$ is a number of order unity which may be determined in term of the (known) present value of the ratio $\lambda_{p} / \lambda_{s}$ [10], and $I$ is the $k$-independent quantity

$$
I=\int_{1}^{\eta_{1} / \eta_{s}} \frac{d \eta}{\eta_{s}} \frac{(g / a)^{2}}{\left(g_{s} / a_{s}\right)^{2}} \sim 1+z_{s}^{-3}\left(g_{1} / g_{s}\right)^{2}
$$

The r.m.s. perturbation amplitude over a comoving length scale $k^{-1}$ (see for instance [6]) is given, in general, by $\left|\delta h_{k}(\eta)\right| \simeq k^{3 / 2}\left|h_{k}\right|=(g / a) k^{3 / 2}\left|\psi_{k}\right|$. For $\eta>\eta_{1}$, we then find

$$
\left|\delta h_{k}(\eta)\right| \simeq \frac{H_{1} a_{1}}{a(\eta) M_{p}}\left(\frac{k}{k_{s}}\right)^{1 / 2} \frac{g_{s}}{g_{1}} z_{s}\left[1+\frac{1}{2} \ln \left(\frac{k_{s}}{k}\right)+z_{s}^{-3}\left(\frac{g_{1}}{g_{s}}\right)^{2}\right]
$$

Equation (2.10) is the main result of this section and we will use it subsequently to estimate the required sensitivity for detection.

It is worth stressing that the leading term in $\left|c_{-}\right|$comes from the integral appearing in eq. (2.4), associated with the freezing of the momentum variable conjugate to $h$. This unusual result is due to the fact [3] that, in the Einstein frame, the scale factor is $a_{E}=a / g$, and our background corresponds to a contracting, rather than expanding, Universe. The amplification of tensor perturbations in a contracting Universe was first considered long ago in 11, 12].

In spite of the presence of a high-curvature regime, we expect our estimates to be valid for scales that went out of the horizon in the dilaton-driven phase, since they follow from the general physical principle that a perturbation and its canonically conjugate momentum 
should remain frozen while outside the horizon. The perturbations thus evolve in a purely kinematical way, giving rise to the logarithmic term in (2.8) from evolution during the dilaton-dominated phase and to the second term $I$ from the stringy epoch.

Finally, let us digress a moment to show the stability of the result (2.10) with respect to $O(d, d)$ transformations which connect [13 different homogeneous string cosmologies. A simple derivation of this nice feature is obtained by working in cosmic time and by using directly the amplitude $h$ [defined in eq. (2.1)] rather than the canonically normalized perturbation $\psi$. It is straightforward to check that, in arbitrary $O(d, d)$-related Bianchi I-type backgrounds (including possible dynamical internal dimensions and an antisymmetric tensor $B_{\mu \nu}$ ), the Fourier modes of $h$ satisfy the following simple equation (see also [9])

$$
\ddot{h_{\omega}}-\dot{\bar{\phi}} \dot{h_{\omega}}+\omega^{2} h_{\omega}=0
$$

where dots denote derivatives with respect to cosmic time and $\bar{\phi}=\phi-\ln \left|\operatorname{detg}_{\mu \nu}\right|^{1 / 2}$ is the so-called shifted dilaton, invariant under $O(d, d)$ transformations. Using the fact that, in any dilaton-driven vacuum cosmology, $\bar{\phi} \sim-\ln t$, we easily obtain, for perturbations well outside the horizon,

$$
h_{\omega}(t) \sim \ln \left|\frac{t}{t_{e x}}\right| \sim \ln |\omega t|,
$$

showing that the spectrum of GW is independent of the chosen string-cosmology background, and increasing our confidence that eq. (2.10) is indeed the generic GW spectrum of a large class of string cosmology models. Note, incidentally, that this is not the case for the electromagnetic perturbations discussed in [14].

\section{Observability}

In order to discuss the observability of our signal it is useful to rewrite our main result (2.10) in terms of present, red-shifted proper frequencies $\omega=k / a$. One easily finds

$$
\begin{gathered}
\left|\delta h_{\omega}\right| \simeq \sqrt{\frac{H_{0}}{M_{s}}} z_{e q}^{-1 / 4} g_{s} z_{s}\left(\frac{\omega}{\omega_{s}}\right)^{1 / 2}\left[1+\frac{1}{2} \ln \left(\frac{\omega_{s}}{\omega}\right)+z_{s}^{-3}\left(\frac{g_{1}}{g_{s}}\right)^{2}\right], \omega<\omega_{s} \\
\omega_{s}=k_{s} / a \simeq z_{e q}^{-1 / 4} \sqrt{H_{0} M_{s}} z_{s}^{-1} \equiv z_{s}^{-1} \omega_{1} \sim z_{s}^{-1} g_{1}^{1 / 2} 10^{11} H z
\end{gathered}
$$


where $z_{e q}=a / a_{e q} \sim 10^{4}$ takes into account the transition from radiation to matter dominance at $t=t_{e q}, \omega_{1}=H_{1} a_{1} / a \sim 10^{11} \mathrm{~Hz}$ is the maximal frequency reached during the string phase, $M_{s}=\lambda_{s}^{-1} \sim H_{1}$, and $H_{0} \sim 10^{-18} \mathrm{~Hz}$ is the present value of the Hubble scale.

It is also convenient to rewrite our results in terms of another commonly used quantity, the fraction of critical density, $\Omega_{G W}=\rho_{G W} / \rho_{c}$, stored in our GW per logarithmic interval of $\omega$. Defining $d \Omega_{G W} /(d \ln \omega)=\omega^{4}\left|c_{-}\right|^{2} /\left(M_{p} H\right)^{2}$ we have

$$
\frac{d \Omega_{G W}}{d \ln \omega}=z_{e q}^{-1} g_{s}^{2}\left(\frac{\omega}{\omega_{s}}\right)^{3}\left[1+\frac{1}{2} \ln \left(\frac{\omega_{s}}{\omega}\right)+z_{s}^{-3}\left(\frac{g_{1}}{g_{s}}\right)^{2}\right]^{2} \sim\left(\frac{\omega}{H_{0}}\right)^{2}\left|\delta h_{\omega}\right|^{2}, \omega<\omega_{s}
$$

It emerges from eq. (3.3) that $\omega_{s}$ plays the role of an effective temperature in the sense that, below $\omega_{s}$, the spectrum is Planckian (up to logarithms of $\omega$ ). The normalization of the spectrum, however, is different from Planck's because of the further amplification due to the stringy phase. Also, we do not expect the spectrum to stay Planckian above $\omega_{s}$ (see below), but rather to keep growing and to reach its maximum at $\omega_{1}$ before falling exponentially.

Finally we give, with the appropriate caveats, the generalization of the above results to frequencies whose exit occurred during the stringy phase, i.e. to the high frequency part $\omega_{s}<\omega<\omega_{1}$. We are aware of the possible dangers in using field theoretic methods to discuss perturbations in this regime. However, in the absence of a full string theoretic calculation, we shall present our results as an indication of what a possible outcome might be. One finds, after straightforward calculations,

$$
\begin{gathered}
\left|\delta h_{\omega}\right| \simeq g_{1} \sqrt{\frac{H_{0}}{M_{s}}} z_{e q}^{-1 / 4}\left[\left(\frac{\omega}{\omega_{1}}\right)^{2-\beta}+\left(\frac{\omega}{\omega_{1}}\right)^{\beta-1}\right] \\
\frac{d \Omega_{G W}}{d \ln \omega} \simeq g_{1}^{2} z_{e q}^{-1}\left[\left(\frac{\omega}{\omega_{1}}\right)^{6-2 \beta}+\left(\frac{\omega}{\omega_{1}}\right)^{2 \beta}\right], \quad \omega_{s}<\omega<\omega_{1}
\end{gathered}
$$

where $\beta=-\log \left(g_{s} / g_{1}\right) / \log z_{s}$ is also the average value of $\dot{g} /(g H)$, which we have assumed to vary little during the string phase. We have also used the fact that during the string phase the curvature stays controlled by the string scale $\lambda_{s}$ so that, in the String frame, the metric describes a de Sitter-like expansion with $z_{s}=a_{1} / a_{s}=\eta_{s} / \eta_{1}$ (see [15] for further details, and for a different derivation of the same spectrum in the Einstein frame).

We would like to discuss now the prospects of observing our spectrum in gravitational wave detectors. Our main emphasis will be on the planned large interferometers LIGO [16] 
and VIRGO [17], which are expected [8] to start operating at sensitivities (for detection of a stochastic GW background) of $d \Omega_{G W} / d \ln \omega=10^{-6}$ in a frequency band around a few hundred $\mathrm{Hz}$, and have set the ambitious final sensitivity goals of $d \Omega_{G W} / d \ln \omega=10^{-10}$ in a frequency band around $\omega_{I}=100 \mathrm{~Hz}$. It may well be, especially in the first stages of operation, that coincidence experiments between bars and interferometers [18] could also be able to reach similar sensitivities at frequencies around $1 \mathrm{KHz}$. We will mention later other possible devices which seem to have some good potential sensitivity, especially in the higher frequency range, but which have not yet matured into concrete operating systems. Detection of stochastic GW backgrounds at frequencies below $1 \mathrm{~Hz}$ does not seem accessible with current technologies, and we therefore limit our attention to the range above $1 \mathrm{~Hz}$.

We are interested in finding the regions in our $\left\{z_{s}, g_{s}\right\}$ parameter space that may be accessible to experimental detection. From eq. (3.2) we can immediately see that the accessible region requires large values of $z_{s}$. We may distinguish values of $z_{s}$ in the range $z_{s}<10^{9}$ (i. e. $\omega_{s}>\omega_{I}$ ), in which the observable spectrum at $\omega_{I}$ comes mainly from perturbations that crossed the horizon during the dilaton-driven era, from those in the range $z_{s}>10^{9}\left(\omega_{s}<\omega_{I}\right)$ in which the observable spectrum comes mainly from those perturbations that crossed the horizon during the stringy era. The predictions in the range $z_{s}>10^{9}$ should be considered as less robust than those in the range $z_{s}<10^{9}$. In addition, we may distinguish values of $g_{s}$ in the range $g_{s} \lesssim g_{1}$ in which the dilaton does not change much during the stringy phase, from those in the range $g_{s} \ll g_{1}$ in which the dilaton changes by a large amount during the stringy epoch.

In all cases we have to impose the bound following from pulsar-timing measurements [19], which implies $d \Omega_{G W} / d \ln \omega \lesssim 10^{-6}$ at $\omega_{P}=10^{-8} \mathrm{~Hz}$. We also accept the bound $\Omega_{G W} \lesssim 0.1$, imposed by standard nucleosynthesis [20]. Moreover, we have derived the spectrum in the linear approximation, expanding around a homogeneous background. We have thus to impose, for consistency, that the amplified perturbations have a negligible back-reaction on the metric, namely $d \Omega_{G W} / d \ln \omega<1$ at all frequencies and times. This, together with the nucleosynthesis bound, requires a range of parameters corresponding to a spectrum which is growing also in the stringy phase, $0<\beta<3$, namely $\left(g_{s} / g_{1}\right)<1$ and $\left(g_{s} / g_{1}\right)>z_{s}^{-3}$. 
Inserting the appropriate numbers in eqs. (3.3), (3.5) we find, respectively, the following conditions for detectability in interferometers, i. e. $d \Omega_{G W} / d \ln \omega>10^{-10}$ at $\omega_{I}=10^{2} \mathrm{~Hz}$ (assuming that the design goals would actually be achieved),

$$
z_{s}^{3} g_{s}^{2}\left[11-\frac{1}{2} \ln z_{s}+z_{s}^{-3}\left(g_{s} / g_{1}\right)^{-2}\right]^{2}>10^{21},
$$

for $z_{s}<10^{9}$, and either

$$
\log _{10} \frac{g_{1}}{g_{s}}<\left(\frac{1}{3}+\frac{1}{18} \log _{10} g_{1}^{2}\right) \log _{10} z_{s}, \quad \beta<3 / 2
$$

or

$$
\log _{10} \frac{g_{1}}{g_{s}}>\left(\frac{8}{3}-\frac{1}{18} \log _{10} g_{1}^{2}\right) \log _{10} z_{s}, \quad \quad \beta>3 / 2
$$

for $z_{s}>10^{9}$.

It may be useful to list approximate forms of the GW spectral distribution, $d \Omega_{G W} / d \ln \omega$, in different regions of parameter space, which we do in Table 1,

\begin{tabular}{|c||c|c|}
\hline & $z_{s}<10^{9}$ & $z_{s}>10^{9}$ \\
\hline \hline$\beta<3 / 2$ & $z_{e q}^{-1} g_{s}^{2}\left(\frac{\omega}{\omega_{s}}\right)^{3}$ & $z_{e q}^{-1} g_{1}^{2}\left(\frac{\omega}{\omega_{1}}\right)^{2 \beta}$ \\
\hline$\beta>3 / 2$ & $z_{e q}^{-1} z_{s}^{-6} g_{1}^{4} g_{s}^{-2}\left(\frac{\omega}{\omega_{s}}\right)^{3}$ & $z_{e q}^{-1} g_{1}^{2}\left(\frac{\omega}{\omega_{1}}\right)^{6-2 \beta}$ \\
\hline
\end{tabular}

Table 1. Approximate forms of $\left(d \Omega_{G W} / d \ln \omega\right)$ in various regions of parameter space, $\omega_{s}=\omega_{1} / z_{s}, \beta=$ $-\log \left(g_{s} / g_{1}\right) / \log z_{s}$.

Actually, if one considers also the amplification of electromagnetic (EM) perturbations in this scenario [14, one finds an even stronger bound following from the condition $\Omega_{E M}<1$, i.e. $\left(g_{1} / g_{s}\right)<z_{s}^{2}$. Such a constraint washes out completely the allowed region of parameter space corresponding to the lower row of Table 1 . The condition $\Omega<1$ has to be imposed, however, for the validity of the linear approach, but it could be evaded in a more general, inhomogeneous model of background, in which the evolution of fluctuations is treated nonperturbatively.

In Figure 1 the allowed region of parameter space, corresponding to the possible detection of the spectrum appearing in the upper row of Table 1, and compatible with the various bounds on the parameters, is plotted by taking $g_{1}=1$ as a reference value. Also shown are 
the parameter intervals in which other detectors may be useful, for the range corresponding to the upper left corner of Table 1.

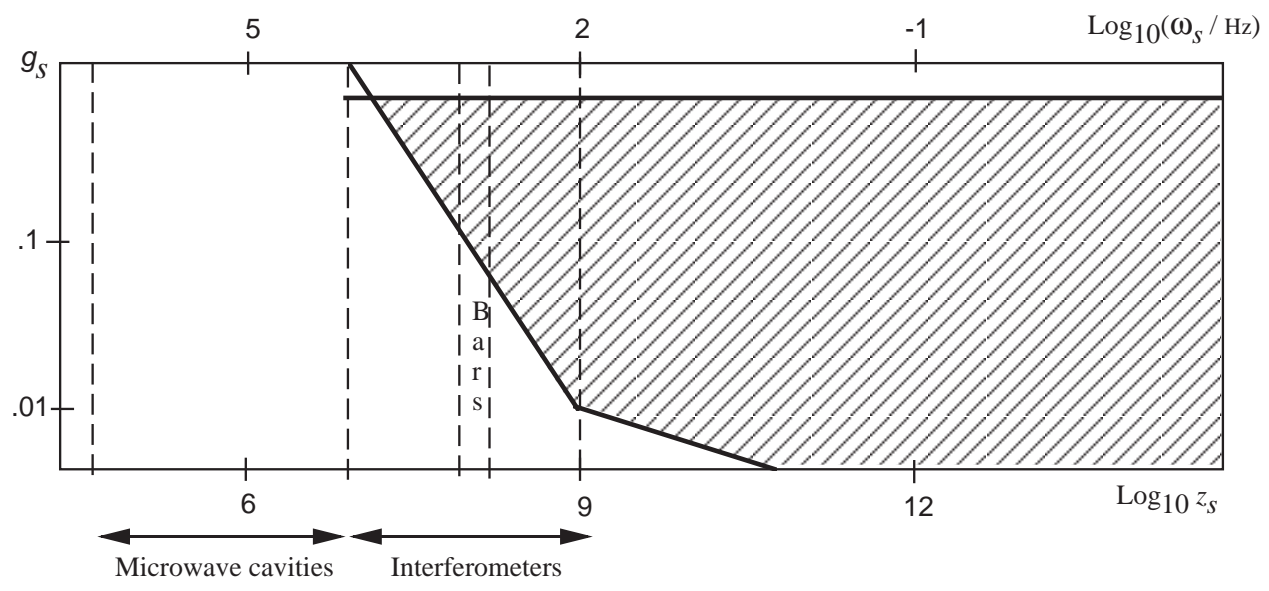

Figure 1. The allowed region in $\left\{z_{s}, g_{s}\right\}$ parameter space corresponding to the first row in Table 1 is the shaded region defined by the various constraints. The dashed lines mark the regions in which various detectors may be useful, for the range $z_{s}<10^{9}$ and $\beta<3 / 2$.

We now turn to discuss in more detail the observability of our spectrum as function of frequency, limiting our attention, for sake of simplicity, to the frequencies leaving the horizon during the dilaton-driven phase [eqs.(3.1), (3.3)]. This spectrum is pictorially described in Fig. 2 for the case $\beta<3 / 2$, in terms of the quantity $\left|\delta h_{\omega}\right|$ (denoted $h_{c}$ in [8]), which represents the characteristic amplitude of a stochastic background. The odd-shaped region in Fig. 2 shows detection sensitivities for the so called "Advanced LIGO" project, in terms of the quantity $h_{3 / y r}$ defined as the amplitude necessary for detection of a stochastic background at the $90 \%$ confidence level in a 1/3 of a year (see [8] for exact definitions). In Fig. 2 we can observe clearly that larger amplification goes together with larger red-shift for this region of parameter space. For a given red-shift $z_{s}$, the higher amplitudes, for all regions of parameter space, are at higher frequencies.

In addition to interferometers and bars, microwave cavities may be operated as gravity wave detectors for the high frequency range $10^{6}-10^{9} \mathrm{~Hz}$. For the $\mathrm{MHz}$ range specific suggestions 21, 22] were actually implemented [23]. As can be seen from Fig. 2, the required sensitivity for detection of gravity waves in the $\mathrm{MHz}$ region is $\left|\delta h_{\omega}\right| \sim 10^{-26}$, corresponding 
to $h_{3 / y r}$ of the same order. We leave to experts to study whether or not such a sensitivity is accessible with current technologies and may be reached in a near future, with microwave cavity detectors or with other experimental apparatus.

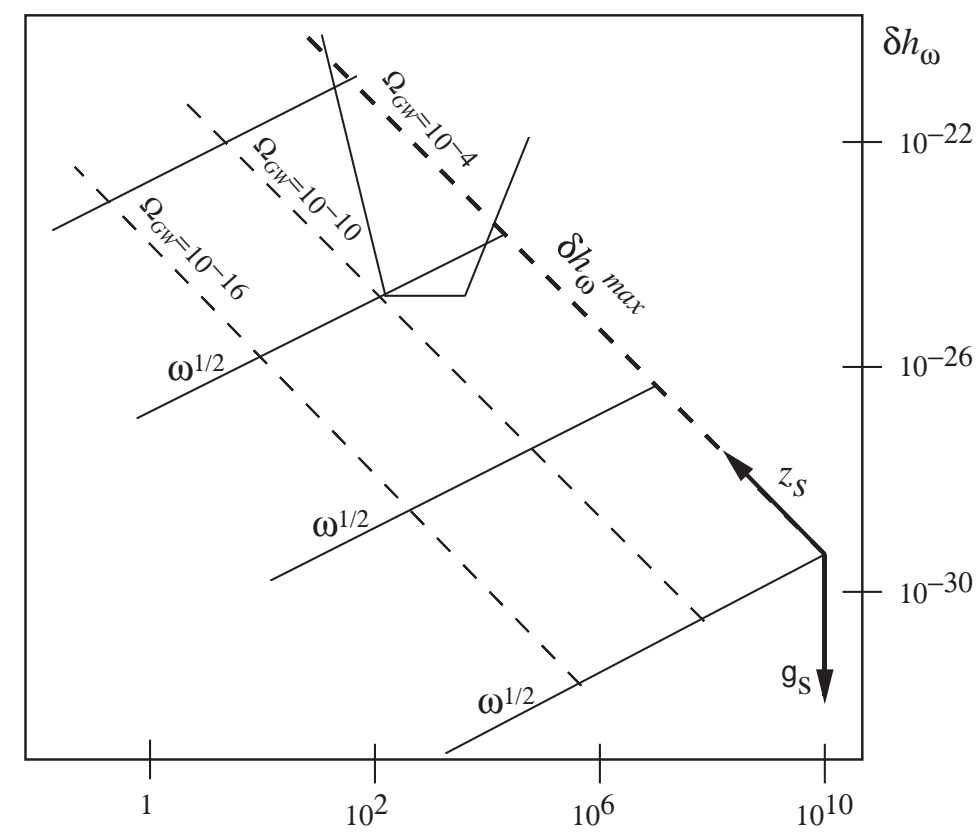

Frequency in Hertz

Figure 2. The characteristic spectral amplitude of gravitational waves $\left|\delta h_{\omega}\right|$. The solid lines show several individual spectra for different values of $z_{s}$ and $g_{s}=1$. The thick dashed line shows the maximum amplitude $\left|\delta h_{\omega}^{\max }\right|$ as a function of $z_{s}$ for $g_{s}=1$. The dashed lines are lines of fixed $g_{s}$ and therefore lines of constant energy density. $\Omega_{G W}$ is roughly the maximal amount of gravitational energy density at a given value of $g_{s}$. Also shown in the figure is the odd-shaped region marking the sensitivity goals for the detection of a stochastic background according to "Advanced LIGO" project.

\section{Conclusion}

We showed that a rather generic string cosmology scenario leads, naturally, to the production of an amplified quasi-thermal spectrum of gravitons during the dilaton-driven phase. This spectrum is very stable under modifications of the background and in particular under $O(d, d)$ transformations. The slope of the spectrum may change for modes crossing the horizon in the subsequent string phase, but remains in general characterized by an enhanced production 
of high frequency gravitons, irrespective of the particular value of the spectral index.

We showed, in particular, that it may be possible to detect such a relic GW background with large interferometers for a range of the two parameters characterizing our class of models. We would like, however, to encourage the study and the developments of gravitational detectors with enhanced sensitivity in the high frequency, $\mathrm{KHz}-\mathrm{GHz}$, range. This frequency band should be in fact all but a "desert" of relic gravitational radiation that one may expect on the grounds of the standard inflationary scenario or from ordinary astrophysical sources. Our string cosmology scenario is unique in predicting a strong signal in this range of frequencies. In general, a sensitivity of $\Omega \sim 10^{-4}-10^{-5}$ (which is not out of reach, in the $\mathrm{KHz}$

region, for coincidence experiments between bars and interferometers [18]), could be already enough to detect a signal, so even a null result at that level of sensitivity would already constrain in a significant way the parameters of the string background, while detection would provide a first glimpse at some new and exciting Planckian physics.

As we stressed, many of our results are independent of details of the string cosmology scenario. However, it would be worthwhile pointing out again that, although some ideas have been put forward [24], a solid string-theoretic treatment of the stringy phase, which we propose as the necessary bridge between the dilaton-driven and the standard decelerated era, does not yet exist. Recent progress 24] on the interpretation of singularities in string theory, as simply a failure to describe physics in terms of the original set of massless fields, may shed light on the resolution of this issue. The understanding of singularities in string theory would certainly help putting our string cosmology scenario on a firmer basis, and may even provide a framework for the calculation of the parameters $g_{s}$ and $z_{s}$.

\section{Acknowledgements}

R. B. is supported in part by an Alon Grant. We would like to thank E. Coccia, S. Finn, P. Michelson, P. Saulson and N. Robertson for discussions about gravity wave detectors. 


\section{References}

[1] G. Veneziano, Phys. Lett. B265 (1991) 287.

[2] M. Gasperini and G. Veneziano, Astropart. Phys. 1 (1993) 317 ; Mod. Phys. Lett. A8 (1993) 3701 .

[3] M. Gasperini and G. Veneziano, Phys. Rev. D50 (1994) 2519.

[4] R. Brustein and G. Veneziano, Phys. Lett. B329 (1994) 429.

[5] N. Kaloper, R. Madden and K. Olive, "Towards a singularity-free inflationary Universe?", Preprint UMN-TH-1333/95 (hep-th/9506027).

[6] R. Brustein, M. Gasperini, M. Giovannini, V. Mukhanov and G. Veneziano, Phys. Rev. D51 (1995), in press.

[7] M. Gasperini and M. Giovannini, Phys. Lett. B282 (1992) 36.

[8] K. S. Thorne, in 300 Years of Gravitation, S. W. Hawking and W. Israel, Eds. (Cambridge Univ. Press, Cambridge, 1987).

[9] M. Gasperini and M. Giovannini, Phys. Rev. D47 (1993) 1519.

[10] V. Kaplunovsky, Phys. Rev. Lett. 55 (1985) 1036.

[11] L. P. Grishchuk, Ann. Acad. Sci. N.Y. 302 (1977) 439.

[12] A. A. Starobinski, Pis'ma Zh. Eksp. Teor. Fiz. 11 (1979) 719.

[13] K. A. Meissner and G. Veneziano, Phys. Lett. B267 (1991) 33; Mod. Phys. Lett. A6 (1991) 3397; M. Gasperini and G. Veneziano, Phys. Lett. B277 (1992) 256.

[14] M. Gasperini, M. Giovannini and G. Veneziano, "Primordial magnetic fields from string cosmology", Preprint CERN-TH/95-85 (hep-th/9504083);

"Electromagnetic origin of the CMB anisotropy in string cosmology", Preprint CERNTH/95-102 (astro-ph/9505041). 
[15] M. Gasperini, "Amplification of vacuum fluctuations in string cosmology backgrounds", in Proc. of the 3rd Colloque Cosmologie (Paris, June 1995), Preprint DFTT-38/95 (hepth/9506140).

[16] A. Abramovici et al., Science 256 (1992) 325.

[17] B. Caron et. al., "Status of the VIRGO experiment", preprint Lapp-Exp-94-15.

[18] P. Astone, J. A. Lobo and B. F. Schutz, Class. Quantum Grav. 11 (1994) 2093.

[19] D. R. Stinebring et al., Phys. Rev. Lett. 65 (1990) 285.

[20] V. F. Schwartzmann, JEPT Lett. 9 (1969) 184;

T. Walker et al, Ap. J. 376 (1991) 51.

[21] F. Pegoraro, E. Picasso, L. Radicati, J. Phys. A11 (1978) 1949.

[22] C. M. Caves, Phys. Lett. B80 (1979) 323.

[23] C. E. Reece et al., Phys. Lett A104 (1984) 341.

[24] E. Kiritsis and K. Kounnas, Phys. Lett. B331 (1994) 51;

A. A. Tseytlin, Phys. Lett. B334 (1994) 315;

E. Martinec, Class.Quant.Grav.12 (1995) 941;

A. Strominger, "Massless black holes and conifolds in string theory", Preprint hepth/9504090;

B. Greene, D. Morrison and A. Strominger, "Black hole condensation and the unification of string vacua", Preprint hep-th/9504145. 